\title{
CHARACTERISTICS OF THERMALLY STIMULATED LUMINESCENCE AND CONDUCTIVITY OF INSULATING CRYSTAL
}

\author{
A. Opanowicz \\ Institute of Physics, Technical University of Łódż \\ Wólczańska 219, 93-005 Łódź, Poland \\ (Received June 23, 1995; revised version October 26, 1995; \\ in final form February 19, 1996)
}

\begin{abstract}
Different characteristics of thermally stimulated luminescence and conductivity in insulator are numerically calculated without the quasi-equilibrium approximation. The results are compared with corresponding results calculated using the quasi-equilibrium approximation. This comparison suggests that the quasi-equilibrium approximation is correct in the low temperature range of the thermally stimulated luminescence and conductivity curves for crystals which have the density of recombination centres higher than $10^{13} \mathrm{~cm}^{-3}$ and the recombination coefficient larger than $10^{-12} \mathrm{~cm}^{3} \mathrm{~s}^{-1}$, or equivalently, for those with the initial free electron lifetime shorter than $10^{-1} \mathrm{~s}$. For the high temperature range of the thermally stimulated luminescence and conductivity curves the quasi-equilibrium approximation gives correct results if the recombination centres density is higher than $10^{14} \mathrm{~cm}^{-3}$ and the recombination coefficient is larger than $10^{-12} \mathrm{~cm}^{3} \mathrm{~s}^{-1}$ or if the initial free electron lifetime is shorter than $10^{-2} \mathrm{~s}$. The results of this paper show also that the so-called "first-order" shape of the thermally stimulated luminescence and conductivity curves is typical not of the weak retrapping only, but it can be obtained also for the strong retrapping.
\end{abstract}

PACS numbers: $78.60 . \mathrm{Kn}, 77.22 . \mathrm{Ej}$

\section{Introduction}

Measured thermally stimulated luminescence (TSL) and thermally stimulated conductivity (TSC) curves are often used for a study of the electron levels in insulating crystals (e.g. $[1,2])$. About 50 different methods of analysis of the TSL and TSC curves have been published (e.g. [1,2]). The formulas for these methods are derived on the basis of the so-called "quasi-equilibrium (QE) approximation" which assumes that [3-8]:

$n \ll h$, 


$$
\left|\frac{\mathrm{d} n}{\mathrm{~d} t}\right| \ll\left|\frac{\mathrm{d} h}{\mathrm{~d} t}\right| \approx\left|\frac{\mathrm{d} a}{\mathrm{~d} t}\right|=\frac{n}{\tau},
$$

where $n$ is the density of thermally released electrons in the conduction band, $h$ is the density of electrons trapped in the active traps, $a$ is the density of holes in the recombination centres, $\tau$ is the free electron lifetime, and $t$ is the time. Condition (2) means that electrons in the conduction band are in quasi-stationary state within the lifetime. The QE assumptions (1) and (2) are, however, not valid for any set of parameters of traps and recombination centres. The QE approximation was examined by several investigators [9-13] which have obtained inconsistent results. Kelly et al. [9] found that for low density of active traps $\left(<10^{15} \mathrm{~cm}^{-3}\right)$ assumptions (1) and (2) are not valid. Shenker and Chen [10] did not find such a restriction. In contradiction to those authors, Lewandowski and collaborators $\{11,12]$ reported that assumption (2) is correct for the case of weak retrapping only. Recently published results of Opanowicz [13] also confirm the invalidity of conditions (1) and (2) for low values of densities of traps and recombination centres $\left(10^{12} \mathrm{~cm}^{-3}\right)$. The above-mentioned authors studied the QE validity for the TSL and TSC curves calculated for different sets of recombination and trapping parameters.

The above-mentioned results suggest possible presence of inaccuracies in calculations using assumptions (1) and (2), in particular, using approximate formulas for calculation of the trap depth (e.g. $[1,2]$ ). A comparison of the results of the TSL and TSC characteristics calculated without and with the QE approximation are, however, not published. In this paper we have presented such a comparison of the TSL and TSC characteristics numerically calculated for different values of the recombination and trapping parameters. For consideration of the TSL and TSC processes an insulator model described by a set of kinetic rate equations is used.

\section{Model and equations for describing the TSL and TSC}

In order to describe TSL and TSC phenomena many authors have used a recombination model of insulator having one kind of active (shallow) traps, one kind of thermally inactive (deep) traps, and one kind of recombination centres. It is assumed that the traps are donor-like and the recombination centres are acceptor-like imperfections. Because it is considered a compensated material, the density of recombination centres is equal to $N=H+M$, where $N$ is the density of recombination centres, $H$ is the density of active (shallow) traps, $M$ is the density of inactive (deep) traps. The equations describing the model for $n$-type insulator (Fig. 1) can be presented in the form (e.g. [3-8]):

$$
\begin{aligned}
& n+h+M=a, \\
& \frac{\mathrm{d} n}{\mathrm{~d} t}+\frac{\mathrm{d} h}{\mathrm{~d} t}=\frac{\mathrm{d} a}{\mathrm{~d} t}=-I=-n \alpha a, \\
& \frac{\mathrm{d} h}{\mathrm{~d} t}=n \beta(H-h)-h s \nu,
\end{aligned}
$$

where $I=n \alpha a$ is the luminescence intensity, $\alpha$ is the recombination coefficient of a free electron with a hole in the recombination centre, $\beta$ is the trapping coefficient 


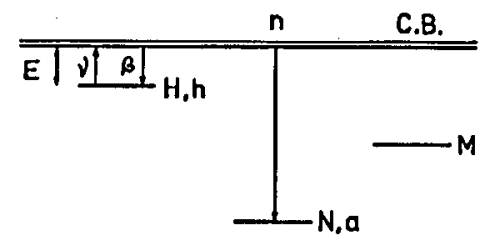

V.B.

Fig. 1. The energy level scheme and electron transitions forming the model which is generally used for interpretation of the TSL and TSC phenomena.

of empty active trap for a free electron, $s=\beta N_{\mathrm{c}}$ is the frequency factor for the active trap, $N_{\mathrm{c}}$ is the density of states in the conduction band, $\nu=s \exp (-E / k T)$ is the probability (per second) of the electron escape from the active trap, $E$ is the active trap depth, $T$ is the temperature, $k=8.617 \times 10^{-5} \mathrm{eV} \mathrm{K}^{-1}$ is the Boltzmann constant. For simplification it is assumed that $\alpha, \beta$, and $s$ are independent of the temperature.

Under assumptions (1) and (2) Eqs. (3)-(5) can be simplified and it is possible to decouple them and derive the equations for the TSL and TSC intensities, the "maximum condition" of the TSL curve, and the equation describing the temperature dependence of active trap occupancy [8]:

$$
\begin{aligned}
& I=-w \frac{\mathrm{d} h}{\mathrm{~d} T}=\frac{n}{\tau}=\gamma h s \nu \\
& n=\frac{h s \nu}{\alpha(M+h)+\beta(H-h)}, \\
& \frac{\alpha\left\{(\alpha-\beta)\left(M+h_{\mathrm{m}}\right) h_{\mathrm{m}}+\left(M+2 h_{\mathrm{m}}\right)\left[\alpha\left(M+h_{\mathrm{m}}\right)+\beta\left(H-h_{\mathrm{m}}\right)\right]\right\}}{\left[\alpha\left(M+h_{\mathrm{m}}\right)+\beta\left(H-h_{\mathrm{m}}\right)\right]^{2}} \\
& \quad=\frac{w E}{s k T_{\mathrm{m}}^{2}} \exp \left(\frac{E}{k T_{\mathrm{m}}}\right), \\
& \frac{\beta}{\alpha}\left(1+\frac{H}{M}\right) \ln \left(\frac{M+h}{M+h_{0}}\right)-\left(1+\frac{\beta H}{\alpha M}\right) \ln \left(\frac{h}{h_{0}}\right) \\
& \quad=\frac{s}{w} \frac{k T^{2}}{E} \exp \left(-\frac{E}{k T}\right) \Sigma(T),
\end{aligned}
$$

where $w=\mathrm{d} T / \mathrm{d} t=\operatorname{const}(T)$ is the heating rate of the sample, $\gamma$ is the fractional recombination probability, $T_{\mathrm{m}}$ is the temperature of the maximum intensity of the TSL (or TSC) curve, $h_{0}$ is the initial density of electrons in the active traps, and where $h_{\mathrm{m}}=h\left(T_{\mathrm{m}}\right)$, and the sum $\Sigma(T)$ is expressed by

$$
\Sigma(T)=\sum_{n=0}^{l}(-1)^{l}(l+1) !\left(\frac{k T}{E}\right)^{l},
$$


where $l$ determines number of terms, e.g. $l=20$ was used in this paper. The formula for the fractional recombination probability $\gamma$ has the form [8]

$$
\gamma=\frac{\alpha a}{\alpha a+\beta(H-h)},
$$

and the free electron lifetime is given by

$$
r=\frac{1}{\alpha a}
$$

where $a=M+h$ when condition (1) is fulfilled. The parameter $\gamma$ shows whether the physical process (either recombination or retrapping) is dominant in the TSL (or TSC) at given temperature. The weak retrapping process (i.e. $\alpha a>\beta(H-h)$ ) corresponds to $0.5<\gamma \leq 1$, whereas the strong retrapping one (i.e. $\alpha a<\beta(H-h)$ ) is described by $0 \leq \gamma<0.5$. If $\gamma \approx 0.5$ there is moderate retrapping (i.e. $\alpha a \approx$ $\beta(H-h)$ ). Equations (6)-(12) were used for numerical calculations of different TSL and TSC characteristics in papers $[8,14,15]$.

\section{Results of calculations and discussion}

In order to examine assumptions (1) and (2) we have solved exactly Eqs. (3)-(5) for the previously studied cases $[8,14,15]$ in which the QE assumptions were used. We have also solved Eqs. (3)-(5) without and with assumptions (1) and (2) for sets of trapping and recombination parameters other than used in $[8,14,15]$. The exact solutions of Eqs. (3)-(5) were found numerically using the Runge-Kutta 4th-order method [16]. The approximate Eqs. (6)-(9) were solved numerically using the Newton method [16]. The calculations were made for a set of parameters: $E=0.4 \mathrm{eV}, \alpha=10^{-12}$ to $10^{-9} \mathrm{~cm}^{3} \mathrm{~s}^{-1}, \beta=10^{-13}$ to $10^{-9} \mathrm{~cm}^{3} \mathrm{~s}^{-1}$, $s=10^{6}$ to $10^{9} \mathrm{~s}^{-1}, H=10^{13}$ to $10^{15} \mathrm{~cm}^{-3}, M=10^{11}$ to $10^{15} \mathrm{~cm}^{-3}$, and two values of initial filling of the traps: $h_{0} / H=10^{-3}$ and 1 , and the value of the heating rate of the crystal $w=0.2 \mathrm{~K} \mathrm{~s}^{-1}$. Different characteristics of the TSL and TSC processes were calculated: $n(T), h(T), \mathrm{d} n(T) / \mathrm{d} t, \mathrm{~d} h(T) / \mathrm{d} t, \mathrm{~d} a(T) / \mathrm{d} t, \gamma(T), \tau(T)$, and the symmetry factor of the TSL (TSC) curve $\mu_{\mathrm{g}}=\left(T_{2}-T_{\mathrm{m}}\right) /\left(T_{2}-T_{1}\right)$, where $T_{1}$ and $T_{2}$ are the temperatures at which the TSL (or TSC) intensity is equal to 0.5 of the maximum intensity, and $T_{2}>T_{1}$.

Figure 2 presents the temperature dependences of the TSL (a) and TSC (b) relative intensities calculated by exact solving of Eqs. (3)-(5) for a set of parameters: $\alpha=10^{-9} \mathrm{~cm}^{3} \mathrm{~s}^{-1}, \beta=10^{-10} \mathrm{~cm}^{3} \mathrm{~s}^{-1}, s=10^{9} \mathrm{~s}^{-1}, H=10^{15} \mathrm{~cm}^{-3}$, $M=10^{13} \mathrm{~cm}^{-3}, N=1.01 \times 10^{15} \mathrm{~cm}^{-3}$, and other parameters as presented above. The shapes of the $I(T) / I_{\mathrm{m}}$ and $n(T) / n_{\mathrm{m}}$ (where $I_{\mathrm{m}}=I\left(T_{\mathrm{m}}\right), n_{\mathrm{m}}=n\left(T_{\mathrm{m}}\right)$ ) curves and other characteristics of these curves are practically identical with the corresponding characteristics calculated using the $\mathrm{QE}$ assumptions in paper [15]. We present three examples of identical values of the TSL characteristics calculated without and with the QE approximation for the case of initial occupancy $h_{0} / H=10^{-3}: T_{\mathrm{m}}=208.679 \mathrm{~K}, T_{2}=218.185 \mathrm{~K}$, and $h_{\mathrm{m}}=4.031 \times 10^{11} \mathrm{~cm}^{-3}$.

Some results of $n, h,|\mathrm{~d} n / \mathrm{d} t|,|\mathrm{d} h / \mathrm{d} t|$, and $|\mathrm{d} a / \mathrm{d} t|=I$ calculated for different temperatures and the parameters of the TSL and TSC of Fig. 2 are presented in Tables IA and IB. Comparing the values of $n$ and $h$ one can see that in both cases of trap occupancy $\left(h_{0}=H\right.$ (Table IA), and $h_{0}=10^{-3} H$ (Table IB)) 

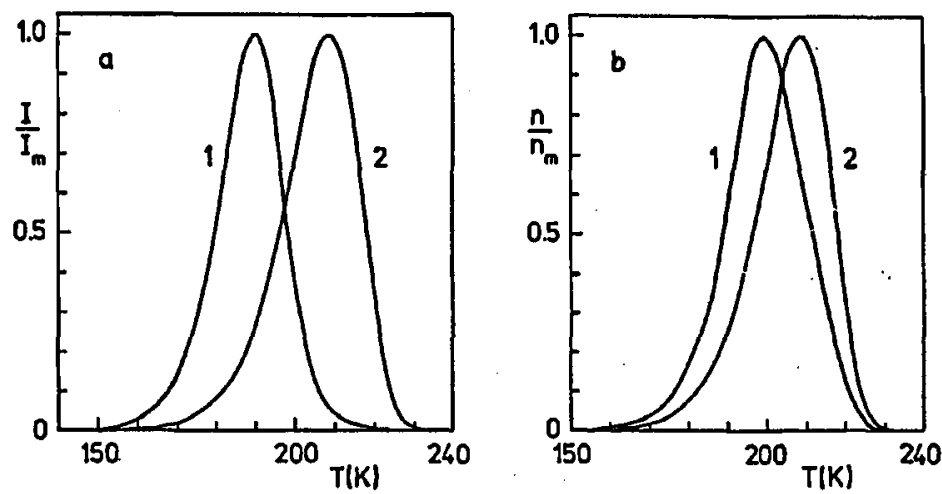

Fig. 2. Temperature dependences of the relative (a) TSL $\left(I / I_{\mathrm{m}}\right)$ and (b) TSC $\left(n / n_{\mathrm{m}}\right)$ intensities calculated by exact solving of Eqs. (3)-(5) for a set of parameters: $E=0.4 \mathrm{eV}$, $\alpha=10^{-9} \mathrm{~cm}^{3} \mathrm{~s}^{-1}, \beta=10^{-10} \mathrm{~cm}^{3} \mathrm{~s}^{-1}, s=10^{9} \mathrm{~s}^{-1}, H=10^{15} \mathrm{~cm}^{-3}, M=10^{13} \mathrm{~cm}^{-3}$, $N=1.01 \times 10^{15} \mathrm{~cm}^{-3}, w=0.2 \mathrm{~K} \mathrm{~s}^{-1}$, and two values of initial occupancy of active traps: (1) $h_{0} / H=1$, and (2) $h_{0} / H=10^{-3}$.

assumption (1) is very well fulfilled $\left(n / h<10^{-4}\right)$. Similarly, the fulfilment of condition (2) is very good ( $\left.|\mathrm{d} n / \mathrm{d} t| /|\mathrm{d} h / \mathrm{d} t|<10^{-4}\right)$ and the approximate Eq. (2) (i.e. $\mathrm{d} h / \mathrm{d} t=\mathrm{d} a / \mathrm{d} t)$ is valid with high accuracy. This suggests that our earlier calcula-

TABLE I

Values of $n, h,|\mathrm{~d} n / \mathrm{d} t|,|\mathrm{d} h / \mathrm{d} t|$, and $|\mathrm{d} a / \mathrm{d} t|$ calculated by exact solving of Eqs. (3)-(5) for different temperatures and the set of parameters of Fig. 2, and the initial trap occupancy (IA) $h_{0} / H=1$, and (IB) $h_{0} / H=10^{-3}$.

\begin{tabular}{c|l|c|c|c|c}
\hline \hline $\begin{array}{c}T \\
{[\mathrm{~K}]}\end{array}$ & $\begin{array}{c}n \\
{\left[\mathrm{~cm}^{-3}\right]}\end{array}$ & $\begin{array}{c}h \\
{\left[\mathrm{~cm}^{-3}\right]}\end{array}$ & $\begin{array}{c}|\mathrm{d} n / \mathrm{d} t| \\
{\left[\mathrm{cm}^{-3} \mathrm{~s}^{-1}\right]}\end{array}$ & $\begin{array}{c}|\mathrm{d} h / \mathrm{d} t| \\
{\left[\mathrm{cm}^{-3} \mathrm{~s}^{-1}\right]}\end{array}$ & $\begin{array}{c}|\mathrm{d} a / \mathrm{d} t| \\
{\left[\mathrm{cm}^{-3} \mathrm{~s}^{-1}\right]}\end{array}$ \\
\hline \multicolumn{5}{c}{$(\mathrm{IA})$} \\
\hline 140 & $3.94 \times 10^{3}$ & $1.00 \times 10^{15}$ & $1.87 \times 10^{2}$ & $3.98 \times 10^{9}$ & $3.98 \times 10^{9}$ \\
160 & $2.49 \times 10^{5}$ & $9.93 \times 10^{14}$ & $9.01 \times 10^{3}$ & $2.49 \times 10^{11}$ & $2.49 \times 10^{11}$ \\
180 & $6.10 \times 10^{6}$ & $8.16 \times 10^{14}$ & $1.70 \times 10^{5}$ & $5.04 \times 10^{12}$ & $5.04 \times 10^{12}$ \\
200 & $3.71 \times 10^{7}$ & $8.21 \times 10^{13}$ & $6.36 \times 10^{4}$ & $3.42 \times 10^{12}$ & $3.42 \times 10^{12}$ \\
220 & $4.87 \times 10^{6}$ & $7.86 \times 10^{11}$ & $2.30 \times 10^{5}$ & $5.25 \times 10^{10}$ & $5.25 \times 10^{10}$ \\
240 & $6.98 \times 10^{-1}$ & $1.93 \times 10^{4}$ & $2.41 \times 10^{-1}$ & $6.98 \times 10^{3}$ & $6.98 \times 10^{3}$ \\
\hline \multicolumn{5}{|c}{$(\mathrm{IB})$} \\
\hline 140 & $3.59 \times 10^{1}$ & $1.00 \times 10^{11}$ & $1.70 \times 10^{0}$ & $3.95 \times 10^{5}$ & $3.95 \times 10^{5}$ \\
160 & $2.26 \times 10^{3}$ & $9.99 \times 10^{11}$ & $8.20 \times 10^{1}$ & $2.49 \times 10^{7}$ & $2.49 \times 10^{7}$ \\
180 & $5.58 \times 10^{4}$ & $9.80 \times 10^{11}$ & $1.56 \times 10^{3}$ & $6.12 \times 10^{8}$ & $6.12 \times 10^{8}$ \\
200 & $5.44 \times 10^{5}$ & $7.23 \times 10^{11}$ & $8.26 \times 10^{4}$ & $5.83 \times 10^{9}$ & $5.83 \times 10^{9}$ \\
220 & $2.91 \times 10^{5}$ & $4.67 \times 10^{10}$ & $1.26 \times 10^{4}$ & $2.93 \times 10^{9}$ & $2.93 \times 10^{9}$ \\
240 & $4.42 \times 10^{-2}$ & $1.22 \times 10^{3}$ & $1.53 \times 10^{-2}$ & $4.43 \times 10^{2}$ & $4.42 \times 10^{2}$
\end{tabular}


tions presented in papers $[8,14,15]$ are made practically without inaccuracies that the $\mathrm{QE}$ assumption can produce.

It is seen from Fig. $2 a$ and $2 b$ that there are some differences between the curves of TSL and TSC calculated for $h_{0} / H=1$ (curves 1). For example, the shape of the TSL curve is rather the "first-order" with $\mu_{\mathrm{g}}=0.44[3,10]$, whereas the TSC curve has typical "second-order" shape with $\mu_{\mathrm{g}}=0.53[3,10]$. The maximum of the TSC curve is shifted towards high temperatures $\left(T_{\mathrm{m}}(\mathrm{TSC})=199.37 \mathrm{~K}\right)$ in relation to the maximum of the TSL curve $\left(T_{\mathrm{m}}(\mathrm{TSL})=189.91 \mathrm{~K}\right)$. These effects can be ascribed to the temperature dependence of the free electron lifetime $\tau$ in this case (e.g. $\tau(T=160 \mathrm{~K})=1.0 \times 10^{-7} \mathrm{~s}$, and $\left.\tau(T=220 \mathrm{~K})=9.3 \times 10^{-5} \mathrm{~s}\right)$. Namely, the increase in $r$ with temperature causes the observed differences between the TSL and TSC curves (e.g. [8]) because of the relation $n=I \tau$ (see Eqs. (4) and (12)). On the other hand, the TSL and TSC curves have almost the same shapes and peak positions if $\tau=\operatorname{const}(T)$. The TSL and TSC curves calculated for $h_{0} / H=10^{-3}$
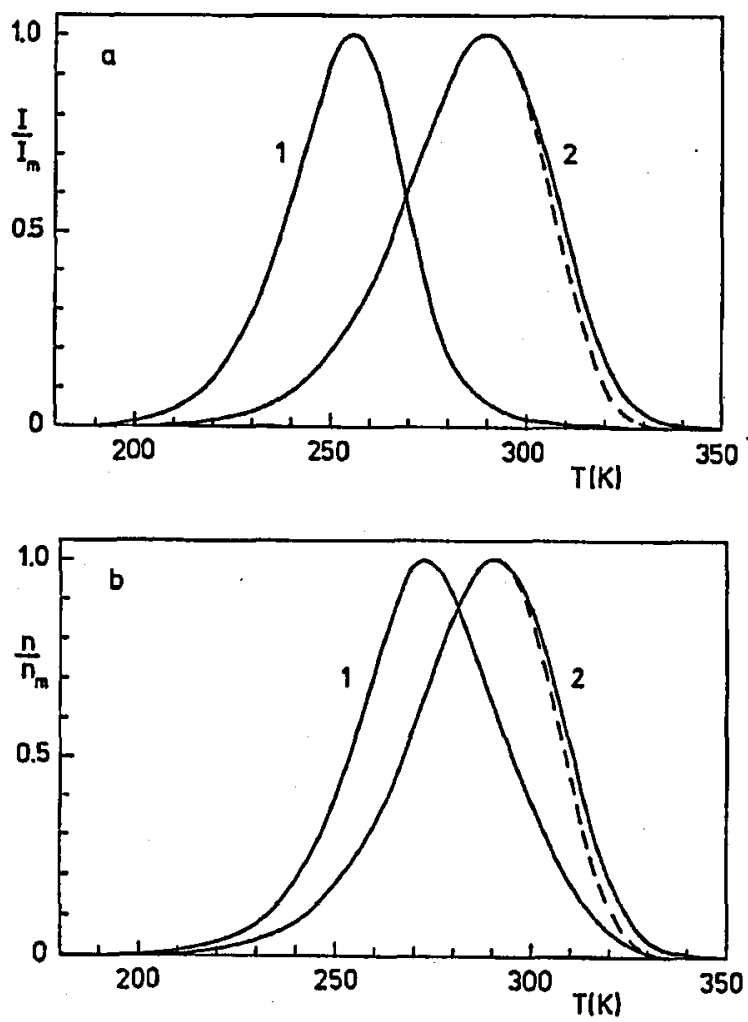

Fig. 3. Temperature dependences of the relative (a) TSL $\left(I / I_{\mathrm{m}}\right)$ and (b) TSC $\left(n / n_{\mathrm{m}}\right)$ intensities calculated without (full lines) and with (dashed lines) the $\mathrm{QE}$ approximation for a set of parameters: $E=0.4 \mathrm{eV}, \alpha=10^{-12} \mathrm{~cm}^{3} \mathrm{~s}^{-1}, \beta=10^{-13} \mathrm{~cm}^{3} \mathrm{~s}^{-1}, s=10^{6} \mathrm{~s}^{-1}$, $H=10^{13} \mathrm{~cm}^{-3}, M=10^{11} \mathrm{~cm}^{-3}, N=1.01 \times 10^{13} \mathrm{~cm}^{-3}, w=0.2 \mathrm{~K} \mathrm{~s}^{-1}$ and two values of initial occupancy of active traps: (1) $h_{0} / H=1$, and (2) $h_{0} / H=10^{-3}$. 
(Fig. $2 \mathrm{a}$ and $2 \mathrm{~b}$, curves 2$)$ illustrate this case $(\tau(T=180 \mathrm{~K}) \approx \tau(T=220 \mathrm{~K}) \approx$ $\left.10^{-4} \mathrm{~s}\right)$.

Figure 3 presents normalized TSL (a) and TSC (b) curves calculated for a set of parameters: $\alpha=10^{-12} \mathrm{~cm}^{3} \mathrm{~s}^{-1}, \beta=10^{-13} \mathrm{~cm}^{3} \mathrm{~s}^{-1}, s=10^{6} \mathrm{~s}^{-1}$, $H=10^{13} \mathrm{~cm}^{-3}, M=10^{11} \mathrm{~cm}^{-3}, N=1.01 \times 10^{13} \mathrm{~cm}^{-3}$, and two different initial trap occupancies: (1) $h_{0} / H=1$, and (2) $10^{-3}$. It is seen from Fig. 3 that both TSL curves and both TSC curves calculated for $h_{0} / H=1$, with and without the $\mathrm{QE}$ approximation, are not resolved.

The values of corresponding TSL and TSC characteristics show, however, small differences, in particular in the high temperature range of the TSL and TSC curves. Values of some TSL and TSC characteristics $\left(T_{m}, T_{11}, T_{22}, h_{m} / h_{0}\right.$, $h_{11} / h_{0}, h_{22} / h_{0}, I_{\mathrm{m}}, n_{\mathrm{m}}, \gamma_{\mathrm{m}}=\gamma\left(T_{\mathrm{m}}\right)$, and $\mu_{\mathrm{g}}$, where $h_{11}=h\left(T_{11}\right)$, and $h_{22}=$ $h\left(T_{22}\right), T_{11}$ and $T_{22}$ are the temperatures at which the TSL (or TSC) intensity

TABLE II

Values of the TSL (IIA) and TSC (IIB) characteristics calculated without (a) and with (b) the QE approximation for the set of parameters used in Fig. 3.

\begin{tabular}{|c|c|c|c|c|}
\hline \multirow[t]{2}{*}{ Characteristic } & \multicolumn{2}{|c|}{$h_{0} / H=1$} & \multicolumn{2}{|c|}{$h_{0} / H=10^{-3}$} \\
\hline & (a) & (b) & (a) & (b) \\
\hline \multicolumn{5}{|c|}{ (IIA) } \\
\hline$T_{\mathrm{m}}[\mathrm{K}]$ & 255.98 & 255.98 & 290.27 & 290.16 \\
\hline$T_{11}[\mathrm{~K}]$ & 196.20 & 196.20 & 215.29 & 215.58 \\
\hline$T_{22}[\mathrm{~K}]$ & 307.83 & 306.89 & 336.49 & 329.92 \\
\hline$h_{\mathrm{m}} / h_{0}$ & $4.459 \times 10^{-1}$ & $4.459 \times 10^{-1}$ & $3.922 \times 10^{-1}$ & $4.102 \times 10^{-1}$ \\
\hline$h_{11} / h_{0}$ & $9.980 \times 10^{-1}$ & $9.980 \times 10^{-1}$ & $9.977 \times 10^{-1}$ & $9.980 \times 10^{-1}$ \\
\hline$h_{22} / h_{0}$ & $1.700 \times 10^{-3}$ & $1.850 \times 10^{-3}$ & $4.345 \times 10^{-4}$ & $5.960 \times 10^{-4}$ \\
\hline$I_{\mathrm{m}}\left[\mathrm{cm}^{-3} \mathrm{~s}^{-1}\right]$ & $5.283 \times 10^{10}$ & $5.295 \times 10^{10}$ & $4.202 \times 10^{7}$ & $4.362 \times 10^{7}$ \\
\hline$\gamma_{\mathrm{m}}$ & $8.919 \times 10^{-1}$ & $8.916 \times 10^{-2}$ & $9.450 \times 10^{-2}$ & $9.436 \times 10^{-2}$ \\
\hline$\tau_{\mathrm{m}}[\mathrm{s}]$ & $2.188 \times 10^{-1}$ & $2.194 \times 10^{-1}$ & 9.585 & 9.606 \\
\hline$\mu_{\mathrm{g}}$ & $4.483 \times 10^{-1}$ & $4.478 \times 10^{-1}$ & $4.493 \times 10^{-1}$ & $4.356 \times 10^{-1}$ \\
\hline \multicolumn{5}{|c|}{ (IIB) } \\
\hline$T_{\mathrm{m}}[\mathrm{K}]$ & 272.84 & 272.98 & 290.94 & 290.81 \\
\hline$T_{11}[\mathrm{~K}]$ & 208.02 & 208.12 & 215.82 & 216.14 \\
\hline$T_{22}[\mathrm{~K}]$ & 331.01 & 326.13 & 336.67 & 330.05 \\
\hline$h_{\mathrm{m}} / h_{0}$ & $9.898 \times 10^{-2}$ & $9.787 \times 10^{-2}$ & $3.780 \times 10^{-1}$ & $3.961 \times 10^{-1}$ \\
\hline$h_{11} / h_{0}$ & $9.913 \times 10^{-1}$ & $9.912 \times 10^{-1}$ & $9.976 \times 10^{-1}$ & $9.978 \times 10^{-1}$ \\
\hline$h_{22} / h_{0}$ & $2.628 \times 10^{-5}$ & $3.400 \times 10^{-5}$ & $4.136 \times 10^{-4}$ & $5.820 \times 10^{-4}$ \\
\hline$n_{\mathrm{m}}\left[\mathrm{cm}^{-3}\right]$ & $2.010 \times 10^{10}$ & $2.036 \times 10^{10}$ & $4.031 \times 10^{8}$ & $4.236 \times 10^{8}$ \\
\hline$\gamma_{\mathrm{m}}$ & $5.519 \times 10^{-1}$ & $5.446 \times 10^{-1}$ & $9.438 \times 10^{-2}$ & $9.424 \times 10^{-2}$ \\
\hline$\tau_{\mathrm{m}}[\mathrm{s}]$ & $9.010 \times 10^{-1}$ & $9.270 \times 10^{-1}$ & 9.598 & 9.615 \\
\hline$\mu_{\mathrm{g}}$ & $5.365 \times 10^{-1}$ & $5.359 \times 10^{-1}$ & $4.470 \times 10^{-1}$ & $4.330 \times 10^{-1}$ \\
\hline
\end{tabular}


is equal to 0.01 of the maximum intensity, and $\left.T_{22}>T_{11}\right)$ calculated with and without the QE approximation for two values of initial trap occupancies $\left(h_{0} / H=1\right.$ and $10^{-3}$ ) and other parameters, as in Fig. 3, are compared in Tables IIA and IIB. One should notice that the case $h_{0} / H=1$ refers to the weak retrapping $\left(\gamma_{m}>0.55\right)$ whereas the case $h_{0} / H=10^{-3}$ corresponds to the strong retrapping process $\left(\gamma_{m} \approx 0.1\right)$. The values of the TSL intensities calculated with and without the QE approximation for $h_{0} / H=10^{-3}$ display considerable differences (Fig. 3a, curves 2). In particular, in high temperature range $\left(T>T_{2}\right)$ the "approximate" $\left(I_{\text {ap }}\right)$ intensity of the TSL is much lower than the "exact" $\left(I_{\mathrm{ex}}\right)$ one (e.g. $I_{\mathrm{ex}} / I_{\mathrm{ap}} \approx$ 2 at $T=320 \mathrm{~K}$, and $\approx 4$ at $T=330 \mathrm{~K}$ ). Similar differences occur between the TSC curves calculated with and without the QE approximation for $h_{0} / H=$ $10^{-3}$ (Fig. 3b, curves 2). The differences between the values of the "exact" and "approximate" TSL characteristics calculated for $h_{0} / H=10^{-3}$ are also observed in Tables IIA and IIB. For example, the "exact" and "approximate" values of $T_{22}$ (TSL) differ by about $6.6 \mathrm{~K}$, and the corresponding values of $h_{22}$ (TSL) differ by about $37 \%$. Similarly, the values of $T_{22}$ (TSC) differ by about $6.7 \mathrm{~K}$, and the values of $h_{22}$ (TSC) differ by about $40 \%$. On the other hand, in the low temperature range $\left(T<T_{\mathrm{m}}\right)$, both the values of TSL and the TSC characteristics calculated with and without the QE approximation show (Table II) only small differences. The differences between "exact" and "approximate" data can be related to the validity of the QE approximation. Tables IIIA and IIIB present the values of $n, h,|\mathrm{~d} n / \mathrm{d} t|$, $|\mathrm{d} h / \mathrm{d} t|$, and $|\mathrm{d} a / \mathrm{d} t|$ calculated for different temperatures and the parameters of the TSL and TSC of Fig. $3 \mathrm{a}$ and 3b. Comparing the values of $n$ and $h$ calculated both for $h_{0} / H=1$ and $10^{-3}$ (Tables IIIA and IIIB) one can see that condition (1) is not fulfilled for $T>300 \mathrm{~K}$. This is because $h(T)$ decreases with increasing temperature faster than $n(T)$ and the ratio of the "initial values" $\left(h_{\mathrm{m}} / n_{\mathrm{m}}\right)_{\mathrm{TSC}}$ (Table II) is not so large as in the case of Fig. 2. For $h_{0} / H=1$ (weak retrapping) condition (2) is almost fulfilled for $T<300 \mathrm{~K}$ and is not realised for $T>300 \mathrm{~K}$. For this case the agreement of the approximate Eq. (2) $(\mathrm{d} h / \mathrm{d} t \approx \mathrm{d} a / \mathrm{d} t)$ is only rough for $T>300 \mathrm{~K}$. For $h_{0} / H=10^{-3}$ (strong retrapping) condition (2) is only roughly valid for $T<300 \mathrm{~K}$, and it is invalid for $T>300 \mathrm{~K}$. For this case similar conlusion can be derived for the approximate $\mathrm{Eq}$. (2) $(\mathrm{d} h / \mathrm{d} t \approx \mathrm{d} a / \mathrm{d} t)$.

Differences between the TSL and TSC curves presented both in Fig. 3a and Fig. 4a can be explained as the effect of variable free electron lifetime above-described in the discussion of the TSL and TSC curves in Fig. 2a.

Comparison of the results for the case of Fig. $2 a$ and $2 b$ (QE valid) with the results for the case of Fig. $3 a$ and $3 b$ (QE valid partly) suggests that the density of recombination centres limiting the $\mathrm{QE}$ validity has the value between $N=10^{13}$ and $10^{15} \mathrm{~cm}^{-3}$. This suggestion is confirmed by the results presented in Fig. $4 \mathrm{a}$ and $4 \mathrm{~b}$ and calculated for the recombination and trap densities greater by one order $\left(N=1.01 \times 10^{14} \mathrm{~cm}^{-3}, H=10^{14} \mathrm{~cm}^{-3}, M=10^{12} \mathrm{~cm}^{-3}\right)$ than the corresponding densities used in the case of Fig. 3a and $3 \mathrm{~b}$. Namely, both TSL and both TSC curves presented in Fig. $4 \mathrm{a}$ and $4 \mathrm{~b}$, and calculated with and without the QE approximation for $h_{0} / H=1$ (weak retrapping), cannot be resolved (e.g. $\left.T_{22 \text { ex }}-T_{22 \text { ap }}<0.1 \mathrm{~K}\right)$. Therefore, there is good agreement between the "exact" and "approximate" data. The "exact" and "approximate" TSL curves calculated 
for $h_{0} / H=10^{-3}$ (strong retrapping) show only small differences at high temperatures (e.g. $T_{22 \mathrm{ex}}-T_{22 \mathrm{ap}}<1 \mathrm{~K}$, Fig. $4 \mathrm{a}$ and $4 \mathrm{a} 1$, curves 2 ). In the temperature range similar properties have also the "exact" and "approximate" TSC curves calculated for $h_{0} / H=10^{-3}$ (Fig. $4 \mathrm{~b}$ and $4 \mathrm{~b} 1$, curves 2). The results presented in Tables IVA and IVB also show that the values of "exact" and "approximate" TSL (and TSC) characteristics are in good agreement. This agreement results from fulfilment of the QE conditions (1) and (2) (see Tables VA and VB). Therefore, the results in Fig. 4 and Tables IV and V show that the density of recombination centres limiting the validity of the QE approximation is of the order $10^{14} \mathrm{~cm}^{-3}$, if the recombination coefficient has the value $10^{-12} \mathrm{~cm}^{3} \mathrm{~s}^{-1}$, i.e. for $N<10^{14} \mathrm{~cm}^{-3}$, and $\alpha<10^{-12} \mathrm{~cm}^{3} \mathrm{~s}^{-1}$ the QE approximation is inaccurate. This conclusion is confirmed by the calculation results of the TSL and TSC characteristics obtained for sets of recombination and trapping parameters (see above) other than those used in Figs. 2 to 4.

TABLE III Values of $n, h,|\mathrm{~d} n / \mathrm{d} t|,|\mathrm{d} h / \mathrm{d} t|$, and $|\mathrm{d} a / \mathrm{d} t|$ calculated by exact solving of Eqs. (3)-(5) for different temperatures and the set of parameters of Fig. 3, and the initial trap occupancy (IIIA) $h_{0} / H=1$, and (IIIB) $h_{0} / H=10^{-3}$.

\begin{tabular}{c|l|l|l|l|l}
\hline \hline $\begin{array}{c}T \\
{[\mathrm{~K}]}\end{array}$ & $\begin{array}{c}n \\
{\left[\mathrm{~cm}^{-3}\right]}\end{array}$ & $\begin{array}{c}h \\
{\left[\mathrm{~cm}^{-3}\right]}\end{array}$ & $\begin{array}{c}|\mathrm{d} n / \mathrm{d} t| \\
{\left[\mathrm{cm}^{-3} \mathrm{~s}^{-1}\right]}\end{array}$ & $\begin{array}{c}|\mathrm{d} h / \mathrm{d} t| \\
{\left[\mathrm{cm}^{-3} \mathrm{~s}^{-1}\right]}\end{array}$ & $\begin{array}{c}|\mathrm{d} a / \mathrm{d} t| \\
{\left[\mathrm{cm}^{-3} \mathrm{~s}^{-1}\right]}\end{array}$ \\
\hline \multicolumn{5}{c}{$(\mathrm{IIIA})$} \\
\hline 200 & $8.21 \times 10^{7}$ & $9.97 \times 10^{12}$ & $1.91 \times 10^{6}$ & $8.29 \times 10^{8}$ & $8.27 \times 10^{8}$ \\
220 & $6.75 \times 10^{8}$ & $9.68 \times 10^{12}$ & $1.29 \times 10^{7}$ & $6.62 \times 10^{9}$ & $6.61 \times 10^{9}$ \\
240 & $3.83 \times 10^{9}$ & $8.01 \times 10^{12}$ & $5.97 \times 10^{8}$ & $3.11 \times 10^{10}$ & $3.11 \times 10^{10}$ \\
260 & $1.43 \times 10^{10}$ & $3.41 \times 10^{12}$ & $1.40 \times 10^{8}$ & $5.06 \times 10^{10}$ & $5.05 \times 10^{10}$ \\
280 & $1.82 \times 10^{10}$ & $4.35 \times 10^{11}$ & $9.18 \times 10^{7}$ & $1.00 \times 10^{10}$ & $1.01 \times 10^{10}$ \\
300 & $7.60 \times 10^{9}$ & $4.53 \times 10^{10}$ & $9.33 \times 10^{7}$ & $1.07 \times 10^{9}$ & $1.16 \times 10^{9}$ \\
320 & $1.20 \times 10^{9}$ & $2.58 \times 10^{9}$ & $3.19 \times 10^{7}$ & $9.26 \times 10^{7}$ & $1.24 \times 10^{8}$ \\
340 & $2.81 \times 10^{7}$ & $2.51 \times 10^{7}$ & $1.37 \times 10^{6}$ & $1.44 \times 10^{6}$ & $2.81 \times 10^{6}$ \\
\hline \multicolumn{5}{|c}{$(\mathrm{IIIB})$} \\
\hline 200 & $7.34 \times 10^{5}$ & $1.00 \times 10^{9}$ & $1.71 \times 10^{4}$ & $9.78 \times 10^{4}$ & $8.08 \times 10^{4}$ \\
220 & $6.06 \times 10^{6}$ & $9.96 \times 10^{9}$ & $1.16 \times 10^{5}$ & $7.82 \times 10^{5}$ & $6.66 \times 10^{5}$ \\
240 & $3.45 \times 10^{7}$ & $9.75 \times 10^{9}$ & $5.42 \times 10^{5}$ & $4.33 \times 10^{6}$ & $3.79 \times 10^{6}$ \\
260 & $1.38 \times 10^{8}$ & $8.80 \times 10^{9}$ & $1.65 \times 10^{6}$ & $1.67 \times 10^{7}$ & $1.51 \times 10^{7}$ \\
280 & $3.43 \times 10^{8}$ & $6.04 \times 10^{9}$ & $1.92 \times 10^{6}$ & $3.84 \times 10^{7}$ & $3.65 \times 10^{7}$ \\
300 & $3.52 \times 10^{8}$ & $2.02 \times 10^{9}$ & $2.19 \times 10^{6}$ & $3.39 \times 10^{7}$ & $3.60 \times 10^{7}$ \\
320 & $7.63 \times 10^{7}$ & $1.64 \times 10^{8}$ & $1.95 \times 10^{6}$ & $5.70 \times 10^{6}$ & $7.65 \times 10^{6}$ \\
340 & $1.84 \times 10^{6}$ & $1.65 \times 10^{6}$ & $9.02 \times 10^{4}$ & $9.42 \times 10^{4}$ & $1.84 \times 10^{5}$
\end{tabular}



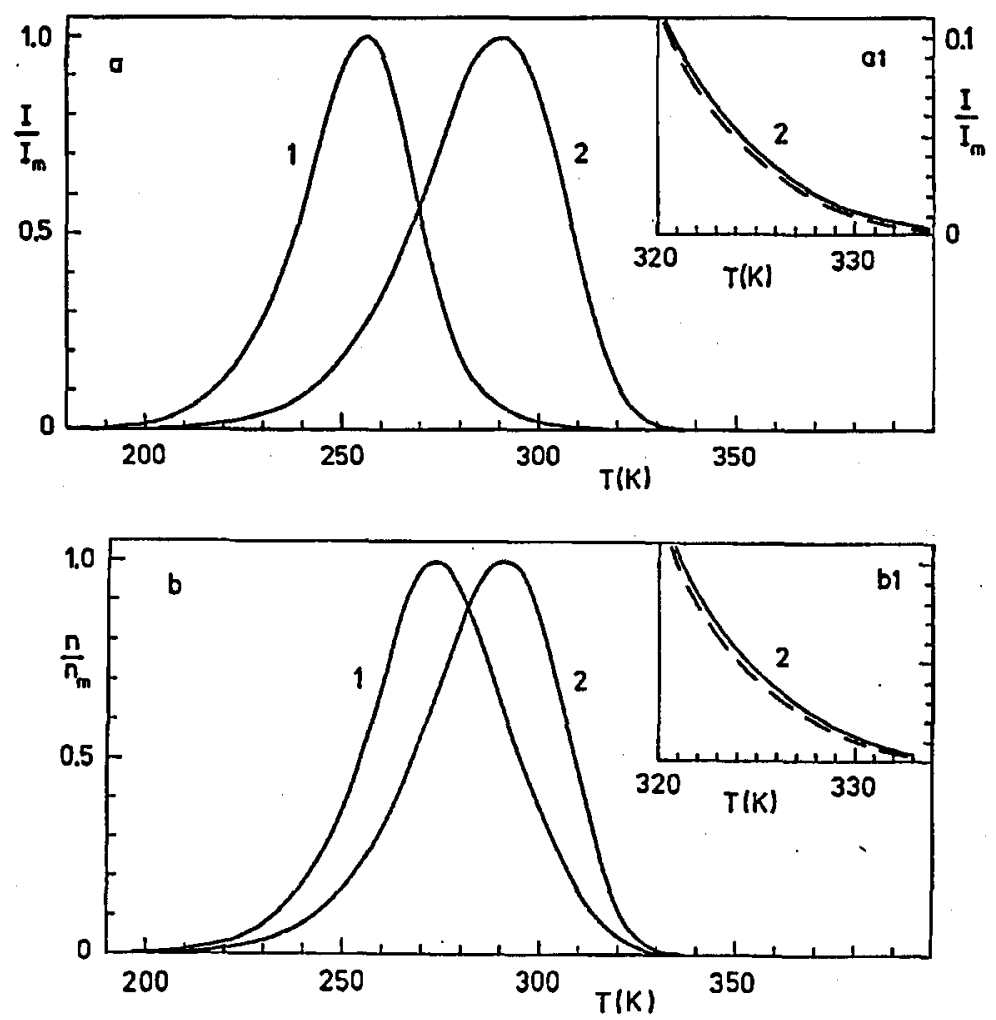

Fig. 4. Temperature dependences of the relative (a) TSL $\left(I / I_{\mathrm{m}}\right)$ and (b) TSC $\left(n / n_{\mathrm{m}}\right)$ intensities calculated without (full lines) and with (dashed lines) the $\mathrm{QE}$ approximation for a set of parameters: $E=0.4 \mathrm{eV}, \alpha=10^{-12} \mathrm{~cm}^{3} \mathrm{~s}^{-1}, \beta=10^{-13} \mathrm{~cm}^{3} \mathrm{~s}^{-1}, s=10^{6} \mathrm{~s}^{-1}$, $H=10^{14} \mathrm{~cm}^{-3}, M=10^{12} \mathrm{~cm}^{-3}, N=1.01 \times 10^{14} \mathrm{~cm}^{-3}, w=0.2 \mathrm{~K} \mathrm{~s}^{-1}$ and two different values of initial occupancy of active traps: (1) $h_{0} / H=1$, and (2) $h_{0} / H=10^{-3}$. Figure $4 \mathrm{al}$ and $4 \mathrm{~b} 1$ show, respectively, the high temperature parts of the TSL and TSC curves in the enlarged scale.

From condition (2) one can see that the free electron lifetime plays important role in reaching the $\mathrm{QE}$ state by electrons in the conduction band because of the relation $|\mathrm{d} n / \mathrm{d} t| \ll n / \tau$. Assuming that the recombination centres are initially fully occupied by the holes and their parameters have the limiting values $N=10^{14} \mathrm{~cm}^{-3}$ and $\alpha=10^{-12} \mathrm{~cm}^{3} \mathrm{~s}^{-1}$, one obtains the initial value of free electron lifetime $\tau_{0}=10^{-2} \mathrm{~s}$. Therefore, the QE approximation is inaccurate for $\tau_{0}>10^{-2} \mathrm{~s}$. On the other hand, Kelly et al. [9] connected the QE validity with the density of active traps. They reported that the QE approximation is invalid if $H<10^{15} \mathrm{~cm}^{-3}$. Their results were, however, obtained for the case assuming absence of deep traps ( $M=0$, i.e. $N=H$ ), which seems to be not fulfilled in real crystals. Shenker and Chen [10] studied the QE validity for the TSL and TSC curves calculated for large values of the recombination coefficients $\alpha>10^{-7} \mathrm{~cm}^{3} \mathrm{~s}^{-1}$. They reported 
that $\mathrm{QE}$ approximation is valid for most of the temperature ranges of interest. On the other hand, Lewandowski et al. [11,12] reported that the QE approximation is valid in whole temperature range of TSL and TSC only in the case of weak retrapping. They, however, calculated the TSL and TSC curves for very low densities of recombination and traps $\left(N\right.$ and $\left.H \leq 10^{6} \mathrm{~cm}^{-3}\right)$. In recent paper Lewandowski et al. [12] present opinion that the QE state and strong retrapping are incompatible. Our results show that their opinion is, in general, not true, i.e. the QE approximation is valid for the case of strong retrapping if $N>10^{14} \mathrm{~cm}^{-3}$ and $\alpha>10^{-12} \mathrm{~cm}^{3} \mathrm{~s}^{-1}$.

TABLE IV Values of the TSL (IVA) and TSC (IVB) characteristics calculated without (a) and with (b) the QE approximation for the set of parameters used in Fig. 4.

\begin{tabular}{|c|c|c|c|c|}
\hline \multirow[t]{2}{*}{ Characteristic } & \multicolumn{2}{|c|}{$h_{0} / H=1$} & \multicolumn{2}{|c|}{$h_{0} / H=10^{-3}$} \\
\hline & (a) & (b) & (a) & (b) \\
\hline \multicolumn{5}{|c|}{ (IVA) } \\
\hline$T_{\mathrm{m}}[\mathrm{K}]$ & 255.98 & 255.98 & 290.17 & 290.16 \\
\hline$T_{11}[\mathrm{~K}]$ & 196.20 & 196.20 & 215.55 & 215.58 \\
\hline$T_{22}[\mathrm{~K}]$ & 306.99 & 306.89 & 330.56 & 329.90 \\
\hline$h_{\mathrm{m}} / h_{0}$ & $1.459 \times 10^{-1}$ & $4.459 \times 10^{-1}$ & $4.119 \times 10^{-1}$ & $4.143 \times 10^{-1}$ \\
\hline$h_{11} / h_{0}$ & $9.980 \times 10^{-1}$ & $9.980 \times 10^{-1}$ & $9.979 \times 10^{-1}$ & $9.980 \times 10^{-1}$ \\
\hline$h_{22} / h_{0}$ & $1.833 \times 10^{-3}$ & $1.850 \times 10^{-3}$ & $6.022 \times 10^{-4}$ & $6.314 \times 10^{-4}$ \\
\hline$I_{\mathrm{m}}\left[\mathrm{cm}^{-3} \mathrm{~s}^{-1}\right]$ & $5.293 \times 10^{11}$ & $5.294 \times 10^{11}$ & $4.386 \times 10^{8}$ & $4.408 \times 10^{8}$ \\
\hline$\gamma_{m}$ & $8.916 \times 10^{-1}$ & $8.916 \times 10^{-2}$ & $9.437 \times 10^{-2}$ & $9.436 \times 10^{-2}$ \\
\hline$\tau_{\mathrm{s}}[\mathrm{s}]$ & $2.193 \times 10^{-2}$ & $2.194 \times 10^{-2}$ & $9.604 \times 10^{-1}$ & $9.602 \times 10^{-1}$ \\
\hline$\mu_{\mathrm{g}}$ & $4.479 \times 10^{-1}$ & $4.478 \times 10^{-1}$ & $4.370 \times 10^{-1}$ & $4.356 \times 10^{-1}$ \\
\hline \multicolumn{5}{|c|}{ (IVB) } \\
\hline$T_{\mathrm{m}}[\mathrm{K}]$ & 272.96 & 272.98 & 290.83 & 290.82 \\
\hline & 208.11 & 208.12 & 216.11 & 216.14 \\
\hline$T_{22}[\mathrm{~K}]$ & 326.61 & 326.13 & 336.69 & 330.05 \\
\hline$h_{\mathrm{m}} / h_{0}$ & $9.798 \times 10^{-2}$ & $9.787 \times 10^{-2}$ & $3.976 \times 10^{-1}$ & $3.979 \times 10^{-1}$ \\
\hline$h_{11} / h_{0}$ & $9.980 \times 10^{-1}$ & $9.912 \times 10^{-1}$ & $9.978 \times 10^{-1}$ & $9.978 \times 10^{-1}$ \\
\hline$h_{22} / h_{0}$ & $3.312 \times 10^{-5}$ & $3.401 \times 10^{-5}$ & $5.753 \times 10^{-4}$ & $6.007 \times 10^{-4}$ \\
\hline$n_{\mathrm{m}}\left[\mathrm{cm}^{-3}\right]$ & $2.033 \times 10^{10}$ & $2.035 \times 10^{10}$ & $4.214 \times 10^{8}$ & $4.216 \times 10^{8}$ \\
\hline$\gamma_{\mathrm{m}}$ & $5.453 \times 10^{-1}$ & $5.446 \times 10^{-1}$ & $9.425 \times 10^{-2}$ & $9.424 \times 10^{-2}$ \\
\hline$\tau_{\mathrm{m}}[\mathrm{s}]$ & $9.243 \times 10^{-2}$ & $9.270 \times 10^{-2}$ & $9.614 \times 10^{-1}$ & $9.611 \times 10^{-1}$ \\
\hline$\mu_{\mathrm{g}}$ & $5.360 \times 10^{-1}$ & $5.359 \times 10^{-1}$ & $4.345 \times 10^{-1}$ & $4.330 \times 10^{-1}$ \\
\hline
\end{tabular}


TABLE V

Values of $n, h,|\mathrm{~d} n / \mathrm{d} t|,|\mathrm{d} h / \mathrm{d} t|$, and $|\mathrm{d} a / \mathrm{d} t|$ calculated by exact solving of Eqs. (3)-(5) for different temperatures and the set of parameters of Fig. 4, and the initial trap occupancy (VA) $h_{0} / H=1$, and (VB) $h_{0} / H=10^{-3}$.

\begin{tabular}{c|l|l|l|l|l}
\hline \hline $\begin{array}{c}T \\
{[\mathrm{~K}]}\end{array}$ & $\begin{array}{c}\text { n } \\
{\left[\mathrm{cm}^{-3}\right]}\end{array}$ & $\begin{array}{c}h \\
{\left[\mathrm{~cm}^{-3}\right]}\end{array}$ & $\begin{array}{c}|\mathrm{d} n / \mathrm{d} t| \\
{\left[\mathrm{cm}^{-3} \mathrm{~s}^{-1}\right]}\end{array}$ & $\begin{array}{c}\mid \mathrm{d} h / \mathrm{d} t] \\
{\left[\mathrm{cm}^{-3} \mathrm{~s}^{-1}\right]}\end{array}$ & $\begin{array}{c}|\mathrm{d} a / \mathrm{d} t| \\
{\left[\mathrm{cm}^{-3} \mathrm{~s}^{-1}\right]}\end{array}$ \\
\hline 200 & $8.23 \times 10^{7}$ & $9.97 \times 10^{13}$ & $1.91 \times 10^{6}$ & $8.29 \times 10^{9}$ & $8.29 \times 10^{9}$ \\
220 & $6.77 \times 10^{8}$ & $9.68 \times 10^{13}$ & $1.29 \times 10^{7}$ & $6.62 \times 10^{10}$ & $6.62 \times 10^{10}$ \\
240 & $3.84 \times 10^{9}$ & $8.01 \times 10^{13}$ & $5.99 \times 10^{7}$ & $3.11 \times 10^{11}$ & $3.11 \times 10^{11}$ \\
260 & $1.44 \times 10^{10}$ & $3.41 \times 10^{13}$ & $1.41 \times 10^{8}$ & $5.06 \times 10^{11}$ & $5.07 \times 10^{11}$ \\
280 & $1.85 \times 10^{10}$ & $4.39 \times 10^{12}$ & $9.11 \times 10^{8}$ & $1.00 \times 10^{11}$ & $1.00 \times 10^{11}$ \\
300 & $7.63 \times 10^{9}$ & $4.57 \times 10^{11}$ & $9.95 \times 10^{7}$ & $1.01 \times 10^{10}$ & $1.12 \times 10^{10}$ \\
320 & $8.27 \times 10^{8}$ & $1.81 \times 10^{10}$ & $2.95 \times 10^{7}$ & $8.13 \times 10^{8}$ & $8.42 \times 10^{8}$ \\
340 & $2.07 \times 10^{6}$ & $1.92 \times 10^{7}$ & $1.86 \times 10^{5}$ & $1.88 \times 10^{6}$ & $2.07 \times 10^{7}$ \\
\hline \multicolumn{5}{|c}{$(\mathrm{VB})$} \\
\hline 200 & $7.48 \times 10^{5}$ & $1.00 \times 10^{9}$ & $1.74 \times 10^{4}$ & $8.40 \times 10^{5}$ & $8.23 \times 10^{5}$ \\
220 & $6.16 \times 10^{6}$ & $9.97 \times 10^{10}$ & $1.18 \times 10^{5}$ & $6.89 \times 10^{6}$ & $6.77 \times 10^{6}$ \\
240 & $3.51 \times 10^{7}$ & $9.78 \times 10^{10}$ & $5.51 \times 10^{5}$ & $3.90 \times 10^{7}$ & $3.85 \times 10^{7}$ \\
260 & $1.41 \times 10^{8}$ & $8.91 \times 10^{10}$ & $1.70 \times 10^{6}$ & $1.56 \times 10^{8}$ & $1.54 \times 10^{8}$ \\
280 & $3.56 \times 10^{8}$ & $6.25 \times 10^{10}$ & $2.06 \times 10^{6}$ & $3.81 \times 10^{8}$ & $3.79 \times 10^{8}$ \\
300 & $3.61 \times 10^{8}$ & $2.08 \times 10^{10}$ & $2.59 \times 10^{7}$ & $3.66 \times 10^{8}$ & $3.68 \times 10^{8}$ \\
320 & $5.19 \times 10^{7}$ & $1.13 \times 10^{9}$ & $1.82 \times 10^{6}$ & $5.01 \times 10^{7}$ & $5.19 \times 10^{7}$ \\
340 & $1.32 \times 10^{5}$ & $1.22 \times 10^{6}$ & $1.18 \times 10^{4}$ & $1.20 \times 10^{5}$ & $1.32 \times 10^{5}$
\end{tabular}

It should be noted also that the TSL and TSC curves (Figs. 2 to 4 , curves 2) calculated for the case of strong retrapping ( $\gamma_{\mathrm{m}} \approx 0.1$ for $h_{0} / H=10^{-3}$ ) have the "first-order" shape with $\mu_{\mathrm{g}}=0.42-0.45$ rather than the "second-order" one characterized by $\mu_{\mathrm{g}}=0.52[1-3]$. Our previous results $[8,14,15]$ calculated with the QE approximation also show that in some cases the "first-order" shape of the TSL and TSC curves can be obtained for the strong retrapping process. Similarly, Shenker and Chen [10] have presented the TSL and TSC curves with $\mu_{\mathrm{g}}=0.42$ calculated for the case of moderate retrapping $\left(\gamma_{\mathrm{m}} \approx 0.5\right)$. Therefore, these results deny conventional opinion (e.g. $[1-3,11,12])$ that the "first-order" shape of the TSL (or TSC) curve is typical of the case of weak retrapping only.

\section{Concluding remarks}

The results of this paper confirm, in principle, the presence of previously found $[9,11,13]$ restriction of validity of the $Q E$ assumption for crystals with low density of imperfections. 
The exact solutions of the kinetic equations describing the TSL and TSC show that the QE conditions $n \ll h$ and $|\mathrm{d} n / \mathrm{d} t| \ll|\mathrm{d} h / \mathrm{d} t|$ are fulfilled in the low temperature range of the TSL and TSC curves if the density of the recombination centres is higher than $10^{13} \mathrm{~cm}^{-3}$ and the recombination coefficient is larger than $10^{-12} \mathrm{~cm}^{3} \mathrm{~s}^{-1}$. On the other hand, in the high temperature range of the TSL and TSC curves the QE conditions are realized if the density of recombination centres is higher than $10^{14} \mathrm{~cm}^{-3}$ and the recombination coefficient is larger than $10^{-12} \mathrm{~cm}^{3} \mathrm{~s}^{-1}$.

Comparison of the results of exact calculations with the results obtained using the QE approximation shows that this approximation yields results with high accuracy if $n / h<10^{-3}$ and $|\mathrm{d} n / \mathrm{d} t| /|\mathrm{d} h / \mathrm{d} t|<10^{-3}$. Accuracy sufficient for rough estimations has the results calculated with the $\mathrm{QE}$ approximation if $n / h<10^{-1}$ and $|\mathrm{d} n / \mathrm{d} t| /|\mathrm{d} h / \mathrm{d} t|<10^{-1}$ only.

The equivalent condition for the $\mathrm{QE}$ approximation, $|\mathrm{d} n / \mathrm{d} l| \ll n / r$, suggests that the free electron lifetime $\tau$ is responsible for occurrence of the QE state in thermally stimulated processes in insulating crystals. Our estimations show that for initial free clectron lifetime $\tau_{0}<10^{-1} \mathrm{~s}$ the QE approximation gives accurate results in the low temperature range of the TSL and TSC curves. For the high temperature range of the TSL and TSC curves the validity of the QE approximation requires fulfilment of the condition $\tau_{0}<10^{-2} \mathrm{~s}$. The above-presented results suggest that before the use of the approximate formulas for evaluating the trap depth in insulating crystal it is necessary to know the value of recombination centres parameters or the value of initial free electron lifetime.

In agreement with the results of our previous papers $[8,14,15]$ the results of this paper show that the TSL and TSC curves corresponding not only to the weak retrapping but also to the strong retrapping process can have the "first-order" shape. Therefore, these results show that the shape of the TSL and TSC curves can give no unique information about the recombination and retrapping processes in insulating crystals.

\section{Acknowledgments}

The author wishes to thank Dr. hab. G. Bak for his help in preparation of the computer program for exact solutions of kinetic equations governing the TSL and TSC processes.

\section{References}

[1] P. Kivits, H.J.I. Hagebeuk, J. Lumin. 15, 1 (1977).

[2] S.W.S. Mc Keever, Thermoluminescence of Solids, Cambridge University Press, Cambridge 1985, Ch. 3.

[3] A. Halperin, A.A. Braner, Phys. Rev. 117, 408 (1960).

[4] G.A. Dussel, R.H. Bube, Phys. Rev. 155, 764 (1967).

[5] I.J. Saunders, J. Phys. C 2, 2181 (1969).

[6] P. Kelly, P. Braunlich, Phys. Rev. B 1, 1587 (1970).

[7] H.J.I. Hagebeuk, P. Kivits, Physica B 83, 289 (1976).

[8] A. Opanowicz, Phys. Status Solidi A 101, 589 (1987). 
[9] P. Kelly, M.J. Laubitz, P. Braunlich, Phys. Rev. B 4, 1960 (1971).

[10] D. Shenker, R. Chen, J. Comp. Phys. 10, 272 (1972).

[11] A.C. Lewandowski, S.W.S. McKeever, Phys. Rev. B 43, 8163 (1991).

[12] A.C. Lewandowski, B.G. Markey, S.W.S. McKeever, Phys. Rev. B 49, 8029 (1994).

[13] A. Opanowicz, Phys. Status Solidi A 148, K103 (1995).

[14] A. Opanowicz, Phys. Status Solidi A 116, 343 (1989).

[15] A. Opanowicz, Phys. Status Solidi A 130, 207 (1992).

[16] A. Marciniak, D. Gregulec, J. Kaczmarek, Basic Numerical Procedures in Turbo Pascal for Your PC, Nakom, Poznań 1992. 